\title{
Urgences
}

\section{La part du mythe dans la relation d'une ville à son support physique : le site de Québec}

\section{Pierre Laplante}

Numéro 17-18, octobre 1987

L'esprit des lieux

URI : https://id.erudit.org/iderudit/025429ar

DOI : https://doi.org/10.7202/025429ar

Aller au sommaire du numéro

Éditeur(s)

Urgences

ISSN

0226-9554 (imprimé)

1927-3924 (numérique)

Découvrir la revue

Citer ce document

Laplante, P. (1987). La part du mythe dans la relation d'une ville à son support physique : le site de Québec. Urgences, (17-18), 148-156.

https://doi.org/10.7202/025429ar d'utilisation que vous pouvez consulter en ligne.

https://apropos.erudit.org/fr/usagers/politique-dutilisation/ 


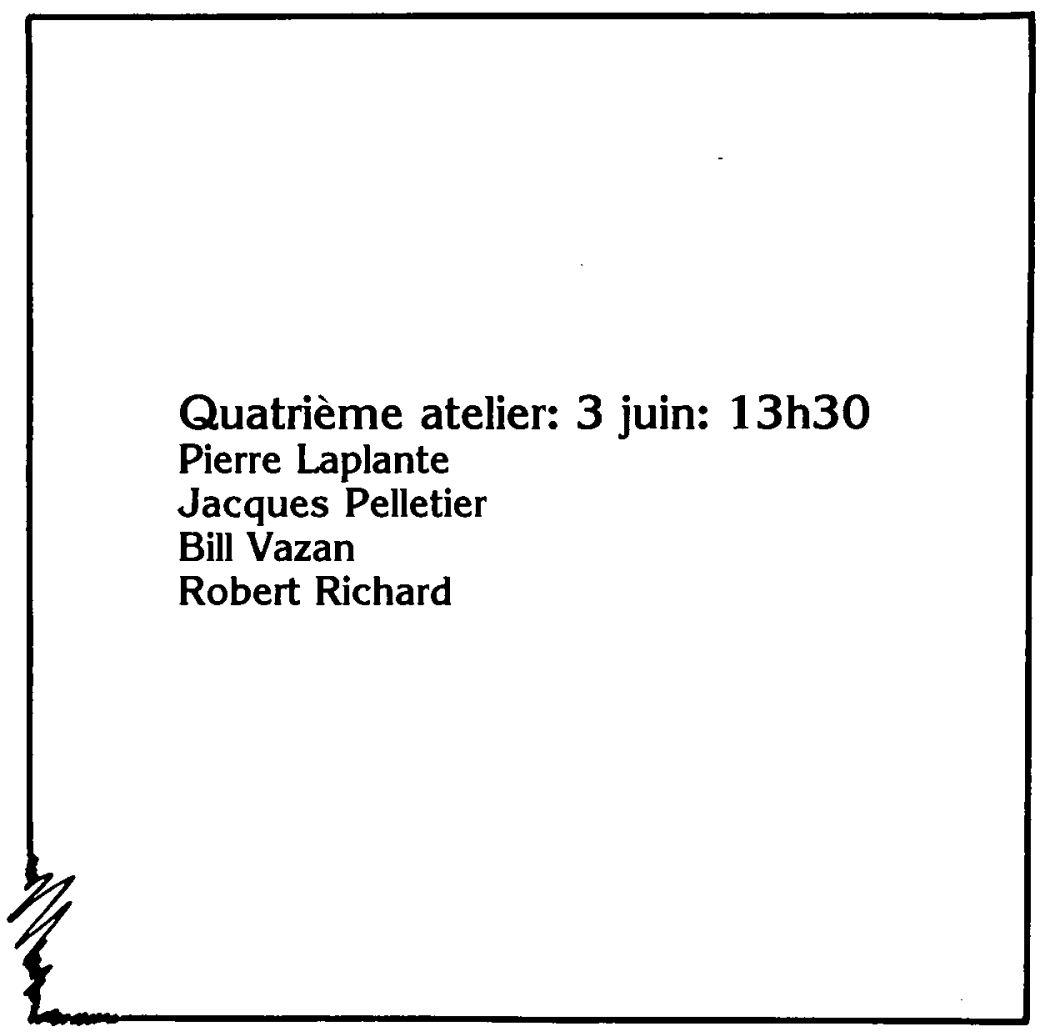




\section{Pierre Laplante}

\section{LA PART DU MYTHE DANS LA RELATION D'UNE VILLE À SON SUPPORT PHYSIQUE: LE SITE DE QUÉBEC}

\section{INTRODUCTION'}

En 1608, Champlain choisissait le Cap Diamant comme site du futur bourg fortifié de Québec afin, apparemment, de protéger le commerce des fourrures des menaces anglaise et iroquoise. Après une description de la géographie physique des lieux, l'auteur remet en question l'interprétation selon laquelle l'érection d'ouvrages militaires sur le site désigné par Champlain découle d'une aptitude naturelle des lieux à la défense ou, plus largement, d'un esprit des lieux. Une lecture responsable des textes d'histoire et de géographie permet en effet d'aller au-delà des apparences de la nécessité et de démontrer que, non seulement l'édification des fortifications a tardé, mais que ces dernières n'ont jamais rempli leur fonction de défense. Ces ouvrages auraient eu une fonction seconde symbolique et mythique. Cette fonction sera retracée à l'aide de textes instaurateurs de modèles d'organisation de l'espace, dont L'Utopie de Thomas More. De la même manière, il sera démontré que la relation d'une ville à son site physique est théorique et langagière. Cette réflexion permettra enfin d'interpréter métaphoriquement le toponyme du Cap Diamant.

\section{DESCRIPTION DU SITE}

À l'échelle de la dizaine de kilomètres, la région de Québec (la consultation du croquis de localisation facilite la lecture de cette partie] présente une configuration de seuil. Géométriquement, le seuil résulte du croisement d'ondulations de signes contraires: le positif faisant saillie au-dessus du négatif en creux. Le croisement se traduit par un relèvement de l'axe négatif et un abaissement de l'axe positif. L'axe négatif correspond dans le concret à la vallée $N E-S W^{2}$ où coule le fleuve, alors que l'axe positif, orienté $N W-S E$, rattache les collines de la Beauce aux collines laurentidiennes. L'axe positif apparaît en surélévation transversale par rapport à la vallée, d'où la notion architecturale de seuil.

Ce sont les variations de dimension du Saint-Laurent et de ses berges qui attirent au premier abord l'attention dans le secteur de Québec. La largeur du fleuve dépasse rarement le kilomètre, face à la ville, entre les sections encore plus étroites des deux ponts et du Cap 
Diamant. En amont et en aval, la largeur peut rapidement excéder les cinq kilomètres. Et le rapprochement des berges s'accompagne toujours d'une augmentation de la profondeur du cours d'eau et de la hauteur des falaises latérales. Ces falaises présentent des pentes raides qui alimentent des éboulis gênant en permanence l'établissement de la végétation. De telles configurations suggèrent que le fleuve franchit un obstacle à Québec, soit ce seuil régional dont il fut question. Ainsi, l'écoulement se concentre à l'endroit où l'obstacle transversal embarrasse le plus; le toponyme algonquin "Québec» rend compte avec justesse de ce rétrécissement de la voie navigable.

L'entaille du Saint-Laurent est escortée d'interfluves tabulaires qui portent la haute-ville de Québec et Lévis à des altitudes pouvant dépasser la centaine de mètres. Ces surfaces se raccordent donc, par-dessus l'entaille du fleuve, permettant ainsi d'établir qu'une surface, primitivement continue, a été entaillée et que les surfaces actuelles sont des lambeaux d'une ancienne plate-forme qui fut appelée "plate-forme de Québec» par Raoul Blanchard. Cette plate-forme supporte l'ensemble des basses terres de la vallée du Saint-Laurent.

Le gonflement de la plate-forme de Québec, selon un axe positif NW-WE, configure le seuil régional. Dans le voisinage de l'axe, les altitudes sont maximales; la surface y porte les hauteurs de Lévis, de la citadelle et de la colline parlementaire; s'y rattachent alors, et avec vigueur, les attributs précédemment notés: profondeur et étroitesse de l'entaille du fleuve, hauteur des falaises aux pentes raides [Cap Diamant]. Le point culminant de la plate-forme, laquelle s'incline doucement vers le nord, porte la citadelle qui domine, par une falaise particulièrement abrupte, la section la plus étroite du Saint-Laurent qui prend des allures de tranchée.

L'interprétation classique propose que l'ancêtre du SaintLaurent se soit enfoncé dans la plate-forme pour y maintenir son cours, au fur et à mesure qu'un mouvement provoquait le bombement transversal de la surface. Les affluents qui n'ont pas la compétence du tronc principal sont déviés vers le SW en amont de Québec [rivières Bécancour et Jacques-Cartier] et vers le NE en aval de Québec [Rivière-du-Sud à Montmagny]. Plus près de l'axe positif, les affluents ne s'imposent pas, ils coulent perpendiculairement, et des chutes sont indicatives de leur lenteur à s'ajuster au relèvement [Chaudière, Etchemin, Saint-Charles, Montmorency].

L'interprétation d'un façonnement exclusivement fluvial est intéressante même si elle surestime largement le travail effectué par l'ancêtre du Saint-Laurent. L'interprétation passe sous silence que 
des glaciers ont occupé ce territoire pendant deux millions d'années et jusqu'à tout récemment, soit il y a dix mille ans. Certaines profondeurs pourraient résulter de surcreusements glaciaires. La tranchée du détroit de Québec, étroite, profonde et ennoyée par l'estuaire, évoque un fjord. Toutefois, le recours au travail des glaciers ou à celui des eaux courantes, pour expliquer certains traits du site, focalise l'attention sur ses traits les plus visuels: le calibrage du fleuve et la vigueur des falaises. Le travail de ces agents est partiel, il s'inscrit dans un paysage déjà fortement configuré par le mouvemement.

Le mouvement de la plate-forme de Québec est générateur des discontinuités qui la morcèlent. La vallée du Saint-Laurent localise une vaste déchirure d'échelle continentale, traversée par une surélévation transversale à Québec. Ce croisement articule un système de couloirs et de tranchées dont certains ont été empruntés et remodelés par les glaciers et l'actuel Saint-Laurent.

En aval de Québec, l'estuaire du Saint-Laurent dessine un biseau, contenant l'île d'Orléans, et pointant vers l'anse de Limoilou. Celle-ci marque l'extrémité orientale d'un large couloir arqué, localisant la basse plaine où méandre la Saint-Charles. Ce couloir, dit de Limoilou, communique vers l'Ouest avec l'étroite tranchée de CapRouge, orientée N-S. Cette tranchée rejoint le couloir arqué de SaintRomuald, occupé par le fleuve face à la ville. Un peu en aval, le couloir est relayé par la tranchée du détroit de Québec, orientée N-S. Enfin, le détroit de Québec rejoint l'anse de Limoilou aux confins de l'estuaire. Le couloir de Limoilou apparaît comme le prolongement de l'estuaire, beaucoup plus que le détroit de Québec, lequel semble contenir un affluent se jetant dans l'estuaire.

Les tranchées et les couloirs qui viennent d'être décrits délimitent la colline de Québec occupée par la haute-ville. Vers l'Est, le recoupement des falaises, à la rencontre du couloir de Limoilou et du détroit de Québec, configure le Cap Diamant, coiffé de la citadelle, à l'extrémité sud des remparts. Couloirs et tranchées insularisent des lambeaux de plate-forme comme la colline de Québec et l'île d'Orléans. De telles relations de formes, dans un espace aussi restreint, inspirent des images évocatrices comme celle qui assimile le Cap Diamant à une porte ouverte surveillant l'entrée du continent.

\section{LA FONCTION PREMIËRE DU SITE}

Il est devenu courant, et de l'ordre du réflexe, d'interpréter le choix du site de Québec par des considérations géopolitiques, lesquelles suggéreraient une aptitude naturelle des lieux à la défense. 
Ainsi, en 1608 , dans le but de protéger le commerce des fourrures des menaces anglaise et iroquoise, Champlain aurait retenu le promontoire du Cap Diamant pour y aménager une forteresse. L'érection d'ouvrages militaires sur le site découlerait d'une vocation inscrite dans la nature. En d'autres termes, il fallait choisir ce site pour se défendre, se défendre puisque le site barrait l'entrée de la voie fluviale navigable.

Cette interprétation du choix de site est déterministe; elle laisse croire que la nature constitue le point d'ancrage de besoins à satisfaire à l'aide de formes construites. C'est tout d'abord en 1603 que Champlain décrivit brièvement le site de Québec sans aucune allusion aux qualités défensives.

Le 22 juin, on jette l'ancre «à Québec qui est un destroict de ladicte riviere de Canadas", toponyme indigène qui signifierait resserrement (...) si les terres étaient cultivées, elles seraient bonnes comme celles de France; comme Cartier, il voit «des diamants dans des rochers d'ardoise, qui sont meilleurs que ceux d'Alençon". Persuadé que Cartier s'était établi en amont, il ne s'amuse à aucun rappel historique; il ne songe non plus à aucun projet d'avenir: le site l'impressionne, c'est tout ${ }^{3}$.

Quelques jours plus tard, ce sera le site de Trois-Rivières qui retiendra l'attention de Champlain du point de vue militaire.

\footnotetext{
Ce seroit à mon jugement un lieu propre pour habiter, $\mathcal{E}$ pourroit-on le fortifier promptement, car sa situation est forte de soy, $\mathcal{E}$ proche d'un grand lac ${ }^{4}$.
}

Jusqu'alors, le site du Cap Diamant avait été délaissé au profit du couloir de Limoilou, où Cartier hiverne en 1535; et de la tranchée de Cap-Rouge, où, en 1541, Cartier tente l'établissement d'une colonie; il sera suivi par Roberval en 1543. En 1608, Champlain place l'Habitation sur la grève au pied du Cap Diamant. Ce n'est qu'en 1618 , soit dix ans plus tard, qu'il reconnaîtra la qualité stratégique du site.

\footnotetext{
Il sera fait un fort composé de cinq bastions à côté de la dite rivière, sur un certain lieu relevé des deux côtés, lequel commandera sur la dite ville et sur le détroit de la dite rivière; de l'autre côté de laquelle et vis-à-vis sera fait un fort de même grandeur pour barrer entièrement le passage de la dite rivière, comme étant l'entrée et la porte du dit pays, sans laquelle on n'y peut aucunement entrer...5
}

Champlain prévoit donc construire une ville à Québec; non pas sur le site du Cap Diamant, ni à son pied près de l'Habitation, mais dans la plaine au sud de la Saint-Charles. Le nom et la composition sont fixés: on mènera à Ludovica 300 familles, chacune composée 
de quatre personnes. Les lois fondamentales seront édictées par le représentant du roi; la justice sera gratuite, sans avocats ni procureurs $^{6}$.

La seule exploitation des richesses de la Nouvelle-France ne nécessitait pas la fondation d'une ville avec forteresse et des remparts. Avant les premiers efforts de colonisation, le golfe du SaintLaurent a été exploité par des pêcheurs espagnols, basques et bretons; leur passage n'a pas laissé d'empreintes majeures dans le paysage. Le projet de Champlain est urbain et social avant d'être simplement militaire et mercantile. La vocation naturelle de défense du site a pris du temps à se manifester, nous venons de le voir, et les premiers équipements militaires seront fragmentaires jusqu'au début du XVIllème siècle?

La fonction militaire a donc connu un retard de démarrage; elle ne s'en remettra pas. Jamais, aussi bien sous le Régime français que sous le Régime anglais, les ouvrages fortifiés ne seront utiles sur le plan militaire. Telle est l'une des conclusions de l'étude menée par Ritchot et alii:

La citadelle, les remparts, les tours Martello, les forts de Lévis, tout cet aménagement militaire n'a jamais servi; il était périmé lors de la conception et il a été dispendieux ${ }^{8}$.

Il découle de l'ensemble de ces considérations une remise en question du rapport présumé des ouvrages fortifiés à leur fonction militaire, et par voie de conséquence, à leur support physique.

\section{LA FONCTION SECONDE}

La fonction militaire, première et évidente qui justifie le choix du site serait contingente. Elle ne découlerait pas d'un rapport incontournable inscrit dans la nature. La fonction seconde des ouvrages fortifiés est discursive; elle se rattache au discours utopien ${ }^{9}$ de la Renaissance qui est en partie mythique, notamment à cause de ses fondements préscientifiques. Le rapport de causalité nature/culture était déjà inscrit dans un livre, celui de L'Utopie. Le choix du site de Québec se serait effectué en fonction d'un discours, d'un texte, celui de L'Utopie de Thomas More. La similitude entre le rocher fortifié de l'Utopie et le site de Québec est marquante. Au début du livre second, Raphaël, voyageur et témoin, dessine le portrait physique d'Utopie:

Mais l'entrée du port est périlleuse, à cause des bancs de sable d'un côté et des écueils de l'autre. A mi-distance environ, se dresse un rocher, trop visible pour être dangereux, sur lequel on a élevé une tour de garde. 
D'autres se cachent insidieusement sous l'eau. Les gens du pays sont seuls à connaître les passes, si bien qu'un étranger pourrait difficilement pénétrer dans le port à moins qu'un homme du pays ne lui serve de pilote. Eux-mêmes ne s'y risquent guère, sinon à l'aide de signaux qui, de la côte, leur indiquent le bon chemin. Il suffirait de brouiller ces signaux pour conduire à sa perdition une flotte ennemie, si importante fut-elle. Sur le rivage opposé se trouvent des criques assez fréquentées. Mais partout un débarquement a été rendu si difficile, soit par la nature, soit par l'art, qu'une poignée de défenseurs suffirait à tenir en respect des envahisseurs très nombreux ${ }^{10}$.

La conjonction entre traits naturels et traits culturels est exemplaire. Il y a relation directe de cause à effet entre le milieu naturel et le milieu bâti. L'île est le résultat d'une prouesse technique du héros fondateur, Utopus, qui l'a séparée du continent. La nature est produite par Dieu; l'homme "choisit» des sites en respectant leur vocation naturelle. D'après Françoise Choay, L'Utopie de More est un texte instaurateur d'espace.

\begin{abstract}
Allons-nous considérer que l'ensemble des textes instaurateurs d'espace est exclusivement formé par les traités d'architecture et les théories de l'urbanisme? Il apparaît nécessaire d'y inclure une autre catégorie d'écrits, les utopies. (...) On s'aperçoit alors que l'utopie, en tant que catégorie littéraire créée par Thomas More, comporte deux traits communs à tous les écrits de l'urbanisme: l'approche critique d'une réalité présente et la modélisation spatiale d'une réalité à venir. Elle offre, au niveau de l'imaginaire, un instrument de conception a priori de l'espace bâti, le modèle".
\end{abstract}

La conjonction lieu/fonction n'est pas inscrite dans la nature comme un rapport univoque, comme le suggère la thèse déterministe. La conjonction est culturelle car déjà inscrite dans un livre, celui de Utopie. Restait à Champlain à transformer le texte de la conception utopienne en monument urbain fortifié, là où la nature s'était permis d'être particulièrement suggestive. En ce sens, la forme urbaine exprime autre chose qu'une simple transformation de matières à des fins de subsistance, autre chose qu'une seule fonction première. La forme urbaine est éminemment symbolique. C'est cette fonction symbolique seconde, utopienne en l'occurence, qui organise les autres. Ainsi, les remparts peuvent servir à des fins: militaires, démographiques (contrôle), économiques (relance) et touristiques.

\title{
LE TOPONYME
}

Un autre texte instaurateur de la Renaissance italienne [Filarète, Trattato d'architettura, 1451-65], propose un récit merveilleux qui raconte l'étape du choix du site d'une ville. Il est rapporté par Françoise Choay qui propose de le considérer avec attention: 
Le «seigneur» de Filarète ayant désiré compléter Sforzinda par un port qui sera nommé Plusiapolis, l'architecte se met en quête d'un site propice et, au lieu élu, découvre, enfoui dans la terre, un mystérieux coffre de pierre. Une fois ouvert, celui-ci livre des vases, des joyaux, et surtout un Livre d'Or, écrit en grec, qu'il faudra faire traduire, et qui relate pour la postérité comment une ville superbe et son port furent, en des temps très anciens, élevés par un prince sur ce site ${ }^{12}$.

Le site, comparable à un lieu élu, est choisi pour ses qualités physiques. Toutefois, le rapport entre le fondateur et le lieu physique, rapport homme/nature, est inscrit dans un livre (celui de l'Utopie?). La découverte du livre, enfoui sous le site élu, constitue la preuve matérielle de l'excellence du choix.

Le coffre contient aussi des pierres précieuses. Celles-ci annonceraient métaphoriquement l'impression de richesse que le site élu évoquera chez le fondateur. Dans le sillage de cette interprétation, on pourra comprendre Champlain, s'illusionnant devant des cristaux de quartz, et la consécration, jusqu'à ce jour, de cette illusion dans le toponyme.

L'éminence du Cap Diamant, tel qu'entrevu précédemment, domine le secteur le plus étroit du fleuve du haut d'une falaise abrupte. Sa prétendue vocation de forteresse naturelle est confortée par la comparaison avec des diamants dont la réputation de dureté et de résistance à l'usure du temps est proverbiale. Il faut rappeler ici la comparaison avec Gibraltar:

Au fond, le rôle dè forteresse n'a pas été très brillant; Québec en a surtout imposé par son allure sur la colline, dominant l'estuaire, par la citadelle du Cap Diamant, par cet air de Gibraltar de l'Amérique du Nord, dont on a si souvent parlé ${ }^{13}$.

Le toponyme pourrait également annoncer, toujours sur le mode de la métaphore, qu'une ville dispendieuse [comme des diamants] serait construite sur le site et qu'elle serait elle-même un bijou.

\section{CONCLUSION}

Abordé sous le thème de l'esprit des lieux, ce retour théorique aux temps de la fondation de Québec par Champlain propose une relecture des circonstance du choix du site. Cette relecture a permis de préciser le caractère utopien des fortifications dans le cadre d'un vaste projet urbain et sociétal. La part du mythe aurait également marqué l'appropriation des lieux par l'acte de nommer. La présente élaboration renseigne également sur l'inaptitude des thèses déterministes à expliquer le caractère monumental des ouvrages fortifiés sur 
le Cap Diamant et, plus largement, à comprendre les rapports homme/nature, qui sont fondamentalement des rapports nature/ culture.

Appliquées au cadre élargi de l'ensemble des lieux naturellement ou culturellement produits, ces conclusions révèlent l'illusion utopienne d'un esprit qui serait naturellement généré par les lieux et qui influerait sur sa destinée ou la nôtre. S'il est un esprit des lieux, il est, à l'image de nos représentations mentales, forgé culturellement par le langage.

\section{NOTES}

1. Cet article reprend les conclusions et converge avec des études menées par Gilles Ritchot et ses collaborateurs depuis une vingtaine d'années; les références principales sont reportées ci-dessous.

2. La lettre $* W \times$ désigne l'ouest selon la convention internationale. Elle est proche des prononciations anglaise, française et allemande [westen].

3. M. Trudel: Histoire de la Nouvelle-France I. Les vaines tentatives 1524-1603, Montréal, Fides, 1963, p. 261.

4. Ibid: p. 262.

5. M. Trudel: Champlain, Montréal, Fides, 1956, p. 76.

6. M. Trudel: Histoire de la Nouvelle-France II. Le comptoir 1604-1627, Montréal, Fides 1966, pp. 253-256. La correspondance entre ce plan systématique d'une colonie de peuplement et l'organisation sociale des Utopiens de $L$ 'Utopie de More est prodigieuse.

7. Entre 1690 et 1709 elles se résumeront à un fort dans la haute-ville et une batterie royale dans la basse-ville.

8. Ritchot et alii: *La fonction symbolique des ouvrages fortifiés du Vieux-Québec *, in Cahiers du CRAD, Vol. 8, no 4, CRAD, Université Laval, Québec, p. 114.

9. De préférence à utopiste ou utopique qui connotent le caractère irréalisable d'un projet. Utopien est l'adjectif formé à partir du titre du livre de More. Ce tivre tente de démontrer l'existence 'ailleurs' d'une île appelée - Utopien, laquelle est habitée par les Utopiens.

10. T. More: LCtopie, Traduction de Marie Delcourt, Paris, Flammarion, 1970, pp. 137-138.

11. F. Choay: La règle et le modèle, Paris, Éditions du Seuil, 1980, pp. 14-15.

12. Ibid: p. 216 .

13. R. Blanchard: Le Québec par limage, Montréal, Beauchemin, 1949, p. 62.

\section{AUTRES DOCUMENTS CONSULTÉS}

G. Ritchot: «e contexte urbain de la fonction de capitale à Québec*, in Cahiers du CRAD, Vol. 8 no 2, CRAD, Université Laval, Québec, pp. 75-85.

G. Ritchot, et C. Feltz: Forme urbaine et pratique sociale, Montréal, Le Préambule et Louwain-la-Meuve, Ciaco, 1985

M. Trudel: Champlain, Montréal, Fides, 1956.

M. Trudel: Histoire de la Nouvelle-France III. La seigneurie des Cent-Associës 1627-1663 tome l Les événernents. Montréal, Fides, 1979.

M. Trudel: Histoire de la Nouvelle-France III. La seigneurie des Cent-Associés 1627-1663 tome 2 La société, Montréal, Fides, 1979.

Comité de rénovation et de mise en valeur du Vieux-Québec, Concept général de réaménagement du VieuxQuébec. Québec, 1970

Service hydrographique du Canada, cartes no 1315,1316 et 1333. 


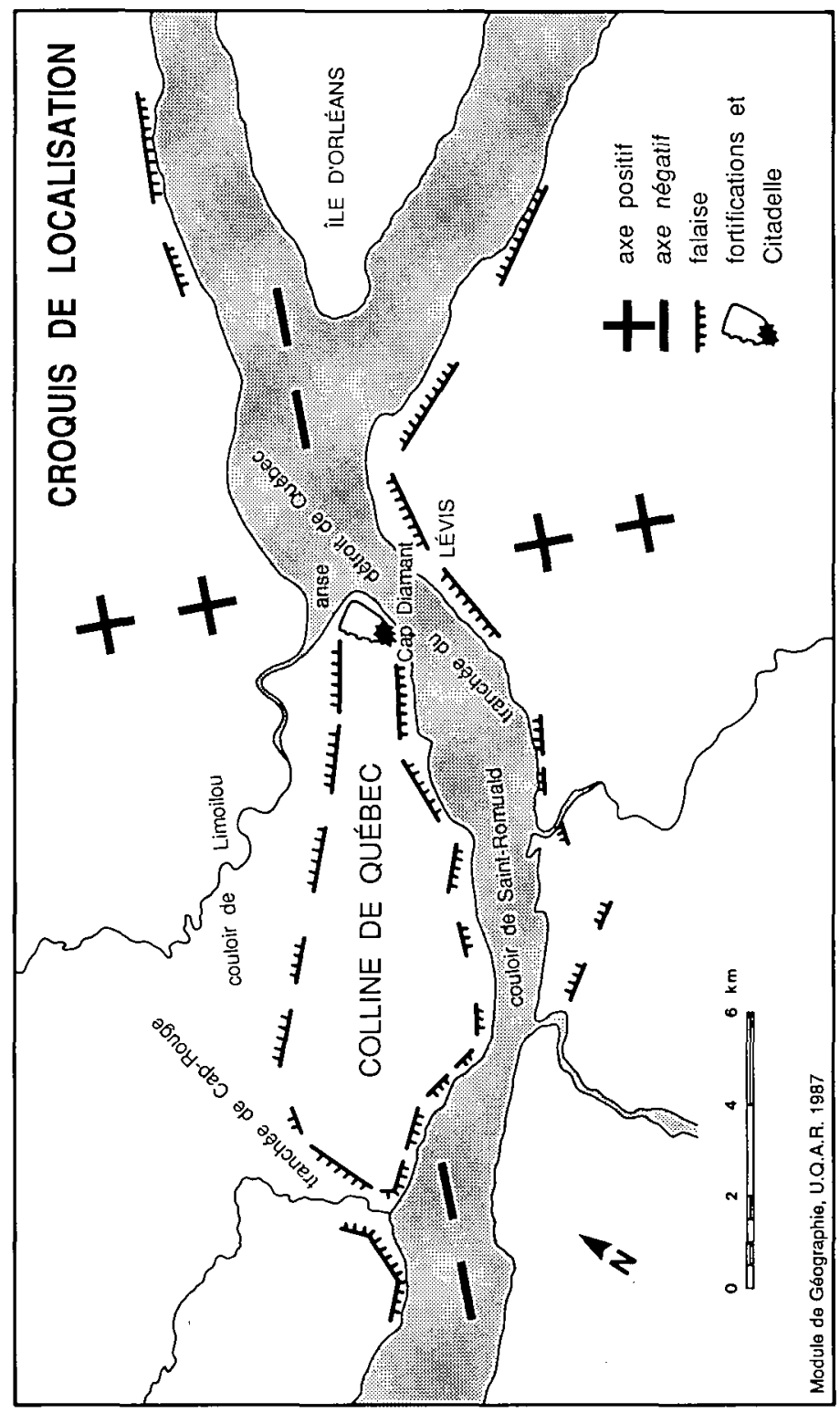

